\title{
Super High Sensitivity Plasmonic Temperature Sensor Based on Square Ring Shape Resonator with Nanorods Defects
}

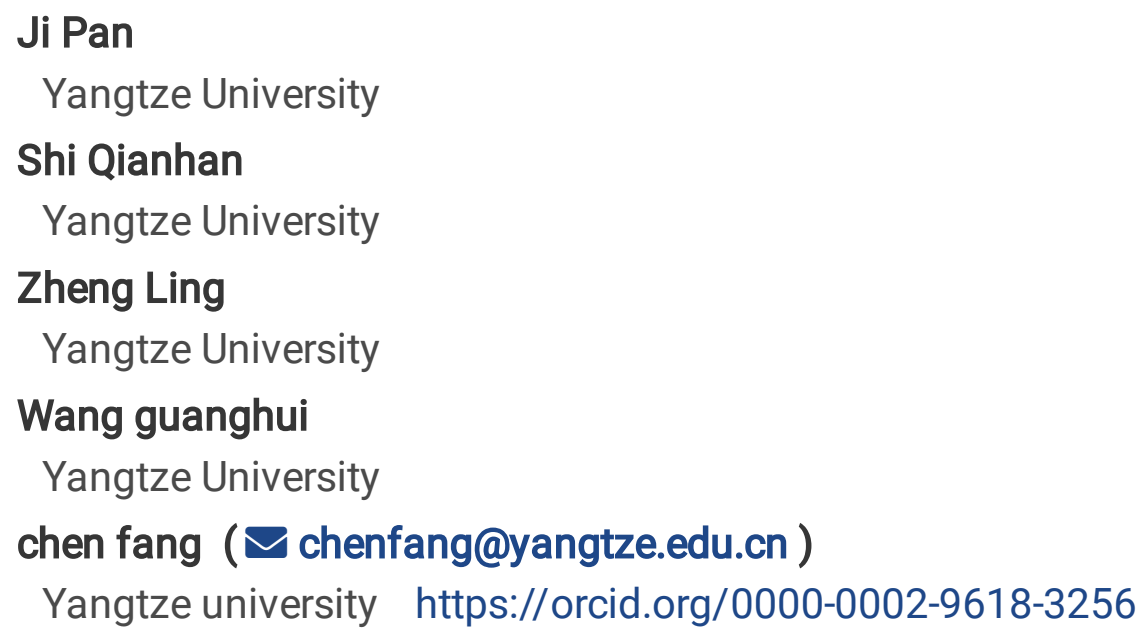

\section{Short Report}

Keywords: Surface plasmon polariton, Metal-insulator-metal, nanorod defect, Refractive index sensor, nanodisk resonator

Posted Date: December 3rd, 2021

DOI: https://doi.org/10.21203/rs.3.rs-1042961/v1

License: (c) (i) This work is licensed under a Creative Commons Attribution 4.0 International License. Read Full License 
Super High Sensitivity Plasmonic Temperature Sensor Based on Square Ring Shape Resonator with Nanorods Defects

Pan Ji, Qihan Shi, Ling Zheng, Guanghui Wang, Fang Chen*

Institute of Quantum Optics and Information Photonics, School of Physics and Optoelectronic Engineering, Yangtze University, Jingzhou 434023, People's Republic of China

\section{*chenfang@yangtzeu.edu.cn (Fang Chen)}

Abstract: A super high sensitivity plasmonic temperature sensor via a metal-insulator-metal (MIM) waveguide system is presented in this paper, the waveguide structure is composed of a square ring shape resonator with nanorods defects and a nanodisk resonator. Finite difference-time domain method (FDTD) is used to study the structure's transmission characteristics and electromagnetic field distributions. Results show that sensitivity will be increased due to the gap plasmonic in the nanorod defect, the nanodisk resonator provides more plasmonic resonant modes for sensing. The positions and intensities of plasmonic resonant modes can be tuned by the radius of nanorod defects and coupling distance. The calculated maximum refractive index and FOM are $4265 \mathrm{~nm} / \mathrm{RIU}$ and 3500, respectively. Compared to the structure without nanorods, the sensitivity is enhanced $33 \%$ for mode 1. For temperature sensing, the proposed structure possesses a relatively high sensitivity of about $1.2 \mathrm{~nm} /{ }^{\circ} \mathrm{C}$. The proposed plasmonic structure provides a basis for designing high sensitivity nano-biosensing, refractive index sensing. 
Key words: Surface plasmon polariton, Metal-insulator-metal, nanorod defect, Refractive index sensor, nanodisk resonator

\section{Introduction}

Surface plasmon polaritons (SPPs) have been widely investigated due to their unique properties of overcoming the traditional diffraction limit [1-5]. In the past few decades, SPPs have been applied in the areas of photonic integrated circuits, plasmonic filters, perfect plasmonic absorbers, plasmonic modulators, optical switches, plasmonic sensors, and modulators [6-10]. Among them, metal-insulator-metal waveguide (MIMW) structures have been proposed and investigated widely due to their low loss, strong field confinement, long propagation distance, and easy fabrication [11-12]. Plasmonic sensors based on MIMW structure have been investigated by many groups, for example, MIM waveguide coupled with an elliptical cavity and a half elliptical groove to produce Fano sensor[13], MIM waveguide coupled with double-ring resonator for refractive index sensing [14]. asymmetric disk resonator for sensing[15].

In the past decade, plasmonic sensors with different configurations have been designed and investigated. For example, in 2020, Chen et al. reported and investigated plasmonic Fano resonance in MIM waveguide coupled to an isosceles triangle resonator [16]. Cui et al. proposed and studied sharp fano resonance based on the coupling between plasmonic stub and circular cavity resonators [17]. Cselyuszka et al. investigated multiple Fano-like MIM plasmonic structure base on triangular resonator for refractive index sensing [18]. Yang et al. proposed concentric rings resonator, with 
a sensitivity of $1060 \mathrm{~nm} / \mathrm{RIU}$ and FOM of 203.8 are achieved [19]. To achieve high sensitivity, much attention has been focused on the structural configuration, system material, and geometrical dimensions, on one hand, the resonance modes are highly sensitive to the environment refractive index and resonator dimensions, but the fabrication complexity is inevitable. On the other hand, the defects often exist in the manufacturing process. How the defects affect the transmission spectrum and the sensing performance, how the defects affect the coupling efficiency between the resonator and the bus waveguide, further research is still needed.

Motivated by the work [20-21], in this paper, multimodes resonance is numerically realized in a compact plasmonic MIM waveguide system. The proposed structure is composed of two bus waveguides, a square ring shape resonator with sixteen nanorods defects, and a middle nanodisk resonator. The introduction of nanorod defects inside the square ring shape resonator can improve the sensitivity by $33 \%$ due to the enhanced gap plasmonic resonance. Results show that the designed plasmonic structure can work as an excellent sensor, a refractive index sensitivity of about $4265 \mathrm{~nm} / \mathrm{RIU}$, and a temperature sensitivity of about $1.2 \mathrm{~nm} /{ }^{\circ} \mathrm{C}$ are obtained in near-infrared band. The results of this paper may have potential application in on-chip multi-wavelength plasmonic nanosensor and multi-band slow light [22-26].

\section{Geometry and Simulation method}

The proposed plasmonic sensor is schematically depicted in Fig. 1, which consists of two MIM waveguides, and a square ring shape resonator with sixteen nanorods 
defects uniformly distributed, a nanodisk resonator is located in the center of the square ring shape resonator.The center of nanodisk and square ring resonator is located in the orgin point. The geometrical parameters are as follows: the outer and inner lengths of the square ring resonator are $L_{1}$ and $L_{2}$, the coupling distance between the square ring resonator and the bus waveguide is $s$. The radius of the nanodisk resonator and the nanorod defect are $R$ and $r$. The width of the bus waveguide is $w=50 \mathrm{~nm}$. The grey and white parts in Fig.1 represent Ag and air, respectively. In the following FDTD simulations, the metal is silver whose frequency-dependent complex relative permittivity is characterized by the Drude model [27-28]:

$$
\varepsilon_{A \mathrm{~g}}(\omega)=\varepsilon_{\infty}-\frac{\omega_{p}^{2}}{\omega^{2}+i \omega \gamma_{p}}
$$

Where $\varepsilon_{\infty}$ is the dielectric constant at the infinite frequency, $\omega_{p}$ and $\gamma_{p}$ stand for the bulk plasmon frequencies and the electron collision, respectively. The parameters for silver can be set as $\varepsilon_{\infty}=3.7, \omega_{p}=9.1 \mathrm{ev}$, and $\gamma_{p}=0.018 \mathrm{ev}[29], \omega$ is the angular frequency of the incident light field. Two-dimensional simulation model is used since the structure in the z-direction is infinite. Perfectly matched layers (PMLs) are applied in the $X$ direction and metal boundary condition was selected in the $\mathrm{Y}$ direction.

Based on the standing wave theory, for the square ring resonator, the resonance wavelength is determined by the equation [30]:

$$
\lambda_{0}=\frac{2 \operatorname{Re}(\text { neff }) L_{\text {eff }}}{k-\phi / 2 \pi}, k=1,2,3 \ldots
$$


Where $\phi$ is the phase shift induced by the reflection, $R \mathrm{e}\left(n_{\text {eff }}\right)$ is the real part of the effective refractive index, and $k$ is the order of the resonance mode. $n_{\text {eff }}$ can be described as: $n_{e f f}(\omega)=\left(\varepsilon_{A g}(\omega)+\left(\frac{k}{k_{0}}\right)^{2}\right)^{1 / 2}$.

According to the coupled-mode theory [31], the transmittance can be calculated by :

$$
T=\left|\frac{\frac{1}{Q_{w}}}{\frac{1}{Q_{w}}+\frac{1}{Q_{r}}+j 2 \delta}\right|^{2}
$$

Where $\delta=\left(\omega-\omega_{0}\right) / \omega_{0}$ is the normalized frequency, $\omega$ is the frequency of the incident spp mode, $\omega_{0}=2 \pi c / \lambda_{0}$, where $\lambda_{0}$ is the resonant wavelength which can be deduced from equation 2. $Q_{r}$ and $Q_{w}$ are the quality factors related to the coupled loss from the square ring resonator into the bus waveguide and the intrinsic loss inside the square ring resonator, respectively.

Fig. 2 shows the transmission spectra of the coupled plasmonic system calculated by FDTD with $w=50 \mathrm{~nm}, s=5 \mathrm{~nm}, R=190 \mathrm{~nm}, L_{1}=500 \mathrm{~nm}$ and $L_{2}=400 \mathrm{~nm}$, $r=18 \mathrm{~nm}$. When there are no nanorod defects, it can be seen five resonances modes (labeled by mode 1, mode 2, mode 3, mode 4, mode 5) of $\lambda_{1}=2603 \mathrm{~nm}, \lambda_{2}=1339 \mathrm{~nm}, \lambda_{3}=913 \mathrm{~nm}, \lambda_{4}=783 \mathrm{~nm}, \lambda_{5}=700 \mathrm{~nm}$. The transmittances of the resonance peaks are $0.35,0.58,0.61,0.77,0.76$, respectively. When nanorod defects are introduced in the square ring resonator, six resonant modes at $\quad \lambda_{1}^{\prime}=3237 \mathrm{~nm}, \lambda_{2}^{\prime}=1621 \mathrm{~nm}, \quad \lambda_{3}^{\prime}=1127 \mathrm{~nm}, \lambda_{4}^{\prime}=896 \mathrm{~nm}, \quad \lambda_{5}^{\prime}=814 \mathrm{~nm}$, $\lambda_{6}^{\prime}=692 \mathrm{~nm}$ can be observed. The transmittances are $0.19,0.49,0.64,0.66,0.51$, 0.62 , respectively. The nanorod defects can create more resonant modes since the gap 
plasmon resonances in the square ring resonator, according to equation 2 , the resonant wavelength $\lambda_{0}$ is proportion to the effective refractive index, the introduction of silver nanorod defects will lead to a larger $n_{\text {eff }}$ and make the resonant wavelength redshift. To investigate the physical mechanism of the resonant resonance, Fig. 3 shows the electric field patterns $|E|$ of the corresponding wavelengths from mode 1 to mode 6 , for mode 5 , we can see that most of the energy is concentrated in the nanodisk resonator, it can be deduced that mode 5 is the nanodisk resonant mode, for the mode $1,2,3,4,6$, the input SPPs are confined in the silver nanorod defects. The gap plasmonic resonance in nanorods and cavity plasmonic resonance offers more flexible control than a popular square ring resonator. Fig. 4 shows the Magnetic field patterns $\left|H_{Z}\right|$ of the corresponding wavelengths from mode 1 to mode 6 . The light spot numbers of $\left|H_{Z}\right|$ patterns in the square ring resonator are 2, 4, 6, 8, 10 for mode $1,2,3,4,6$, respectively. These above characteristics indicate that mode 1, 2, $3,4,6$ are more sensitive to the dimension of silver nanorod defects, while mode 5 is more sensitive to the dimension of the nanodisk resonator. The field enhancement of the modes 1-6 in the gap of nanorod surface will benefit to the design of multichannel sensor.

The influence of dimensions of the nanorod defects $r$ on the transmittance of the proposed structure is then investigated. As shown in Fig. 5, when the radius of nanorod defects increases from $r=12 \mathrm{~nm}$ to $21 \mathrm{~nm}$ with a step of $3 \mathrm{~nm}$, it can be seen that the resonant wavelengths are red-shifted, the increasing $r$ will change the resonant condition and the optical path in the square ring resonator. The transmittance 
peaks are also changed with the increase of $r$, this is because the impedance matching condition is changed when varing $r$. According to equation 3, the Fullwave at half maximum $(\mathrm{FWHM}) \quad F W H M \approx 4 \pi \mathrm{c}\left(1 / Q_{\omega}+1 / Q_{r}\right) / \omega^{2}$ is becoming larger with the increase of $r$, since the separation region between neighboring nanorods is becoming smaller, which is benefit to the gap plasmonic resonance. Therefore, the resonant modes can be tuned by the radius of the nanorod defects.

The effect of coupling distance $s$ on the transmittance of the proposed structure is studied in details. As shown in Fig. 6, when the coupling distance $s$ increases from $s=5 \mathrm{~nm}$ to $20 \mathrm{~nm}$ with a step of $5 \mathrm{~nm}$, it can be seen that the resonant wavelengths are slightly blue-shifted, and the transmittance peaks and FWHM are becoming smaller, the results are in line with the paper [20-21]. Fig. 7 shows the transmission spectra of the plasmonic Fano system with nanodisk radius $R$, when $R$ decreases from $R=195$ to 180 with a step of 5 , it can be seen that the resonant wavelengths are blue-shifted, the transmittance peaks of mode 1, 2, 3, 4 are becoming larger, but the transmittance peak of mode 5 is becoming smaller. The different behaviors are attributed to the different formation mechanisms of mode 5 , when $R$ decreases to less than $175 \mathrm{~nm}$, the mode 5 will disappear since the coupling distance between the nanodisk resonator and the inner square ring resonator is larger than 25 nm.

Finally, we investigated the refractive index sensing characteristics of the proposed structure, changing the medium filled in the white part of the structure in Fig 1 . When the refractive index is increased from 1 to 1.08 with a step of 0.02 , the 
corresponding transmission spectra are shown in Fig 8 (a), it can be seen that the resonant wavelengths have a considerable red-shifts. The refractive index sensitivity $S$ is an important parameter to evaluate the sensor's attributes, it can be defined as $S=d \lambda / d n(n m / R I U)[32]$, where $d \lambda$ is the change of the resonance wavelength and $d n$ is the change of refractive index. Fig. 8 (b) shows the linear relationships between the refractive index and the resonant wavelength of modes 1-6, the sensitivity are approximately $4265 \mathrm{~nm} / \mathrm{RIU}, 1665 \mathrm{~nm} / \mathrm{RIU}, 1110 \mathrm{~nm} / \mathrm{RIU}, 830$ $\mathrm{nm} / \mathrm{RIU}, 785 \mathrm{~nm} / \mathrm{RIU}$, and $680 \mathrm{~nm} / \mathrm{RIU}$ for modes 1-6, respectively. The maximum $S$ of the structure is $4265 \mathrm{~nm} / \mathrm{RIU}$.

The proposed plasmonic waveguide structure can be used for temperature sensor. When the medium of the structure is filled with ethanol material, the refractive index of ethanol material can be calculated by: $n=n_{0}-d n / d T\left(T-T_{0}\right)$ [33], where $T_{0}$ is the ambient temperature at $20^{\circ} \mathrm{C}, d n / d T=3.94 \times 10^{-4}, n_{0}=1.36084$, T is the ambient temperature. As shown in Fig. 9 (a), when the ambient temperature decreases from $T=60^{\circ} \mathrm{C}$ to $-100^{\circ} \mathrm{C}$ with a step of $40^{\circ} \mathrm{C}$, it can be seen that the resonant wavelengths have a red-shifts. Fig. 9 (b) shows the linear relationships between the ambient temperature and the resonant wavelength of modes 1-6. The temperature sensitivity can be defined as $S=d \lambda / d T\left(n m /{ }^{0} C\right)$, where $d \lambda$ is the change of the resonance wavelength and $d T$ is the change of ambient temperature. From Fig. 9 (b), the sensitivity are approximately $1.2 \mathrm{~nm} /{ }^{0} \mathrm{C}$ for mode $1, \quad 0.7 \mathrm{~nm} /{ }^{0} \mathrm{C}$ for mode 2 , $0.5 \mathrm{~nm} /{ }^{0} \mathrm{C}$ for mode $3,0.4 \mathrm{~nm} /{ }^{0} \mathrm{C}$ for mode $4,0.3 \mathrm{~nm} /{ }^{0} \mathrm{C}$ for mode 5, $0.26 \mathrm{~nm} /{ }^{0} \mathrm{C}$ for mode 6 . The maximum $S$ of the structure is $1.2 \mathrm{~nm} /{ }^{0} \mathrm{C}$. The 
sensitivity comparison of the proposed structure and the structure without nanorod defect is shown in Table 1, it can be seen that when introducing the nanorod defect, the sensitivity is increased by $33 \%$ for mode $1,16.7 \%$ for mode $2,25 \%$ for mode 3 , $33 \%$ for mode $4,11 \%$ for mode 5 , respectively. The figure of merit (FOM) is another important parameter for evaluating the sensing performance. It can be calculated by $F O M=d T / d n T$, where $d T$ is the change of the transmittance and $d n$ is the change of refractive index. The FOM distribution of the wavelength is shown in Fig. 9 (c), the calculated maximum FOM is 3500 . Therefore, a remarkably sensitivity and FOM can be achieved for mode 1 with the proposed nanorod defect structure. The sensing performance of other related structures is compared and the results are shown in Table 2. The sensitivity of the present nanorod defect coupled square ring resonator structure is relatively good compared to previous work [].

\section{Conclusion}

In conclusion, we have investigated by FDTD simulation a refractive index and temperature sensor based on a square ring resonator with nanorods defects and a nanodisk resonator. Results show that resonances modes, transmittance peaks, and FWHM can be tuned by the geometrical parameters, such as the radius of silver nanorod defects, coupling distance, the radius of the nanodisk. Based on the enhanced gap plasmon resonance, a refractive index sensitivity of about $4265 \mathrm{~nm} / \mathrm{RIU}$, FOM about 3500 and a temperature sensitivity of about $1.2 \mathrm{~nm} /{ }^{\circ} \mathrm{C}$ were obtained. Sensitivity is increased by $33 \%$ for mode 1 when comparing with the structure without nanorod defect. The designed structure can be applied to the areas of on-chip 
plasmonic nanosensor and the design of multichannel sensor.

\section{Declaration}

\section{Ethical Approval}

Not applicable

\section{Consent to Participate}

Not applicable

\section{Consent to Publish}

The author confirms that the work described has not been published before and has been approved by all co-authors

\section{Ethical Statement}

No conflict of interest exits in the submission of this manuscript, and manuscript is approved by authors for publication. I would like to declare on behalf of my co-authors that the work described was original research that has not been published previously, and not under consideration for publication elsewhere, in whole or in part. Funding: Supported by the Yangtze Fund for Youth Teams of Science and Technology Innovation (Grant No. 2015cqt03) . National Natural Science Foundation of China (Grant No. 11747091). Yangtze Fund for college students' innovation and entrepreneurship.

\section{Competing Interests}

The authors declare that they have no known competing financial interests or personal 
relationships that could have appeared to influence the work reported in the paper.

\section{Availability of data and materials}

Not applicable

\section{References:}

[1]S. Zhang, D.A. Genov, Y. Wang, M. Liu, X. Zhang, Plasmon-induced transparency in metamaterials. Phys. Rev. Lett 101 (2008) 047401.

[2] C. Genet, T.W. Ebbesen, Light in tiny holes. Nature. 445 (2007) 39 .

[3]F. Chen, H. F. Zhang, L. H. Sun, J.J. Li, C.C. Yu, Temperature tunable Fano resonance based on ring resonator side coupled with a MIM waveguide. Opt. Laser. Technol 116 (2019) 293-299 .

[4] H. Raether, Surface plasmons on smooth and rough surfaces and on gratings, Springer, Berlin, Heidlberg, 1998.

[5] Q. Zhang, X.G. Huang, X.S. Lin , J. Tao, X.P. Jin, A subwavelength coupler-type MIM optical filter, Opt. Express 17 (2009) 7549-7554.

[6] G. Wang, H. Lu, X. Liu, Y. Gong, Numerical investigation of an all optical switch in a graded nonlinear plasmonic grating, Nanotechnology 23 (2012) 444009.

[7] H. Bahri, S. Mouetsi, A. Hocini, H.B. Salah, A high sensitive sensor using MIM waveguide coupled with a rectangular cavity with Fano resonance. Opt. Quantum Electron 53 (2021) 332.

[8] M. Singh, S. K. Raghuwanshi, O. Prakash, Modeling of grating assisted hybrid plasmonic filter and its on chip gas sensing application, IEEE sens. J. 19 (2019) 
4039-4044.

[9] J.F. Chen, J.N. Li, X. Liu, S.T. Rohiah, H. Tian, D.W. Qi, Fano resonance in a MIM waveguide with double symmetric rectangular stubs and its sensing characteristics, Opt. Commun 482 (2021) 126563.

[10] S. Khani, M. Dannaie, P. Rezaei, Tunable single mode band pass filter base on metal-insulator-metal plasmonic coupled U shaped cavities, IET Optelectron. 13 (2019) 161-171.

[11] J. Park, H. Kim, B. Lee, High order plasmonic bragg reflection in the metal-insulator-metal waveguide bragg grating, Opt. Express 16 (2008) 413-25.

[12] Y. Neo, T. Matsumoto, T. Watanabe, M. Tomita and H. Mimura, Transformation from plasmon induced transparency ti induced absorption through the control of coupling strength in metal-insulator-metal structure, Opt. Express 24 (2016) 26201:8. [13] R.E. Haffar, A. Farkhsi, O. Mahboub, Optical properties of MIM plasmonic with an elliptical cavity resonator, Applied. Phys. A 126 (2020) 486.

[14] N. Amoosoltani, K. Mehrabi, A. Zarifkar, A. Farmani, N. Yasrebi, Double ring resonator plasmonic refractive index sensor utilizing dual band unidirectional reflectionless propagation effect, Plasmonics 16 (2021) 1277-1285.

[15] S.L. Li, Y. Wang, R.Z. Jiao, L.L. Wang, G.Y. Duan, L. Yu, Fano resonances based on multimode and degenerate mode interference in plasmonic resonator system, Opt. Express, 25 (2017) 3525.

[16] F. Hu, F. Chen, H.F. Zhang, L.H. Sun, C.C. Yu, Sensor based on multiple Fano resonances in MIM waveguide resonator system with silver nanorod-defect, Optik 
$229(2021) 166237$.

[17] B.F. Yun, R.H. Zhang, G.H. Hu, Y.P. Cui, Ultra sharp fano resonances induced by coupling between plasmonic stub and circular cavity resonator, Plasmonic 11 (2016) 1157:1162.

[18] N. Jankovic, N. Cselyuszka, Multiple Fano like MIM plasmonic structure based on triangular resonator for refractive index sensing, Sensors 18 (2018) 287.

[19] Z.J. Zhang, J.B. Yang, X. He, J.J. Zhang, J. Huang, D.B. Chen, Y.X. Han, Plasmonic refractive index sensor with high figure of merit based on concentric rings resonator. Sensor 18 (2018) 116.

[20] C.T.C. Chao, Y.F.C. Chau, H.P.P. Chiang, Highly sensitive metal-insulator-metal plasmonic refractive index sensor with a centrally coupled nanoring containing defects. J. Phys. D 54 (2021) 115301.

[21] Y.F.C. Chau, T.Y. Ming, C.T.C. Chao, R. Thotagamuge, M. R.R. Kooh, H.J. Huang, C.M. Lim, H. P. Chiang, Significantly enhance coupling effect and gap plasmon resonance in a MIM cavity based sensing structure. Sci. Reps 11 (2021) 18515.

[22] F. Chen, H.F. Zhang, L.H. Sun, J.J. Li, C.C. Yu, Temperature tunable Fano resonance base on ring resonator side coupled with a MIM waveguide, Opt Laser Technol, 116 (2019) 293-299.

[23] C.H. Wu, Z.C. Guo, S.X. Chen, J.Yang, K.H. Wen, Refractive index sensing based on multiple Fano resonances in a plasmonic defective ring-cavity system, Results Phys, 27 (2021) 104508. 
[24] Y. Zhang et al. High quality factor multiple Fano resonances for refractive index sensing. Opt. Lett 43 (2018) 1842.

[25] X. Yan, Z. Zhang, 1.j. Liang, M.S. Yang, D.Q. Wei, X.X. Song, H.T. Zhang, Y.Y. Lu, L.H. Liu, M.J. Zhang, T. Wao, J.Q. Yao, A multiple mode integrated biosensor based on higher order Fano metamaterials, Nanoscale. 12 (2020) 1719-1727.

[26] F. Chen, D. Z. Yao, Realizing of plasmon Fano resonance with a metal nanowall moving along MIM waveguide. Opt. Commun. 369 (2016) 72-78.

[27] J. Zhu, N. Li, MIM waveguide structure consisting of a semicircular resonant cavity coupled with a key-shaped resonant cavity. Opt. Express 28 (2020) 19978.

[28] K.Wen, Y. Hu, L. Chen, J. Zhou, M. He, L. Lei, Z. Meng, Y. Wu, J. Li, Fano resonance based on end coupled cascaded ring MIM waveguides structure. Plasmonic 12 (2017) 1875-1880.

[29] P.B. Johnson, R.W. Christy, Optical constants of the noble metal. Phys. Rev. B 6 4370.

[30] Z.F. Li, K.H. Wen, L. Chen, L. Lei, J.Y. Zhou, Refractive index sensor based on multiple Fano resonances in a plasmonic MIM structure. Appl. Opt 58 (2019) 4878-4883.

[31] H. Xu, H.J. Li, B.X. Li, Z.H. He, Z.Q. Chen, M.F. Zheng, Influential and theoretical analysis of nano-defect in the stub resonator. Sci. Reps 6 (2016) 30877.

[32] Y. Chen, Y. Xu, J. Cao, Fano resonance sensing characteristics of MIM waveguide coupled square convex ring resonator with metallic baffle. Results Phys 14 (2019) 102420. 
[33] G.L. Xiao, Y.P. Xu, H.Y. Yang, Z.T. Ou, J.Y. Chen, H.O. Li, X.P. Liu, L.Z. Zeng,

J.Q. Li, High sensitivity plasmonic sensor based on fano resonance with inverted $u$ shaped resonator. Sensors 21 (2021) 1164.

[34] J.Y. Zhang, H.L. Feng, Y. Gao, Plasmonic narrowband filter based on an euilateral triangular resonator with a silver bar. Photonics 8 (2021) 244.

[35] G.L. Lan, Z.X. Jin, J.P. Nong, P. Luo, C.C. Guo, Z.G. Sang, L.Dong, W. Wei, Narrow band perfect absorber based on dielectric metal metasurface for surface enhanced infrared sensing. Appl. Sci 10 (2020) 2295.

[36] M. Bazgir, M. Jalalpour, F.B. Zarrabi, A.S.Arezoomand, Design of an optical switch and sensor based on a MIM coupled waveguide using a DNA composite. J. Electron. Mater 49 (2020) 2173:2178.

[37] Y.L. Wang, S.L. Li, Y.Y. Zhang, L. Yu, Independently formed multiple fano resonances for ultra high sensitivity plasmonic nanosensor. Plasmonic 13 (2018) 107:113.

[38] H.Q. Wang, C.Z. Fan, J.N. He, P. Ding, E.J. Liang, Q.Z. Xue, Double Fano resonances due to interplay of electric and magnetic plasmon modes in planar plasmonic structure with high sensing sensitivity. Opt. Express 21 (2013) 2236. 


\section{Figure captions:}

Fig. 1. The schematic diagram of the proposed plasmonic system composed of two bus waveguides (width $w$ ), a square ring shape resonator (outer length $L_{1}$ and inner length $L_{2}$ ) with sisteen nanorods defects (with radius $r$ ) uniformly distributed. A air nanodisk is located in the center of the square ring shape resonator, the center of nanodisk and square ring resonator are located in orgin point $(0,0)$

Fig.2. The transmission spectra of the coupled plasmonic system with and without sisteen nanorods defects in the square ring resonator. (The geometrical parameters were set as $w=50 \mathrm{~nm}, s=5 \mathrm{~nm}, R=190 \mathrm{~nm}, L_{1}=500 \mathrm{~nm}$ and $\left.L_{2}=400 \mathrm{~nm}, \quad r=18 \mathrm{~nm}\right)$.

Fig.3. Electric field patterns $|E|$ of the corresponding wavelengths from mode 1 to mode 6 , respectively.

Fig.4. Magnetic field patterns $\left|H_{Z}\right|$ of the corresponding wavelengths from mode 1 to mode 6 , respectively.

Fig.5. The transmission spectra of the plasmonic Fano system with different radius of silver nanorod of $r=12,15,18,21 \mathrm{~nm}$.

Fig.6. The transmission spectra of the plasmonic Fano system with different coupling distance of $\mathrm{g}=5,10,15,20 \mathrm{~nm}$. 
Fig.7. The transmission spectra of the plasmonic Fano system with different radius of the nanodisk $\mathrm{R}=180,185,190,195 \mathrm{~nm}$.

Fig.8. (a) The transmission spectra of the proposed plasmonic Fano system with different refractive indexes . (b) Relationships between the refractive index and the resonant wavelengths.

Fig.9. (a)The transmission spectra of the plasmonic Fano system with different ambient temperatures (b) Relationship between the ambient temperature and the resonance wavelengths. (c) The FOM distribution with the wavelength of the structure.

Table 1 . The sensitivity of the proposed plasmonic structures with nanorod defects and without nanorod defects in mode 1-6.

Table 2. Comparison of sensing performance to other related plasmonic structures. 


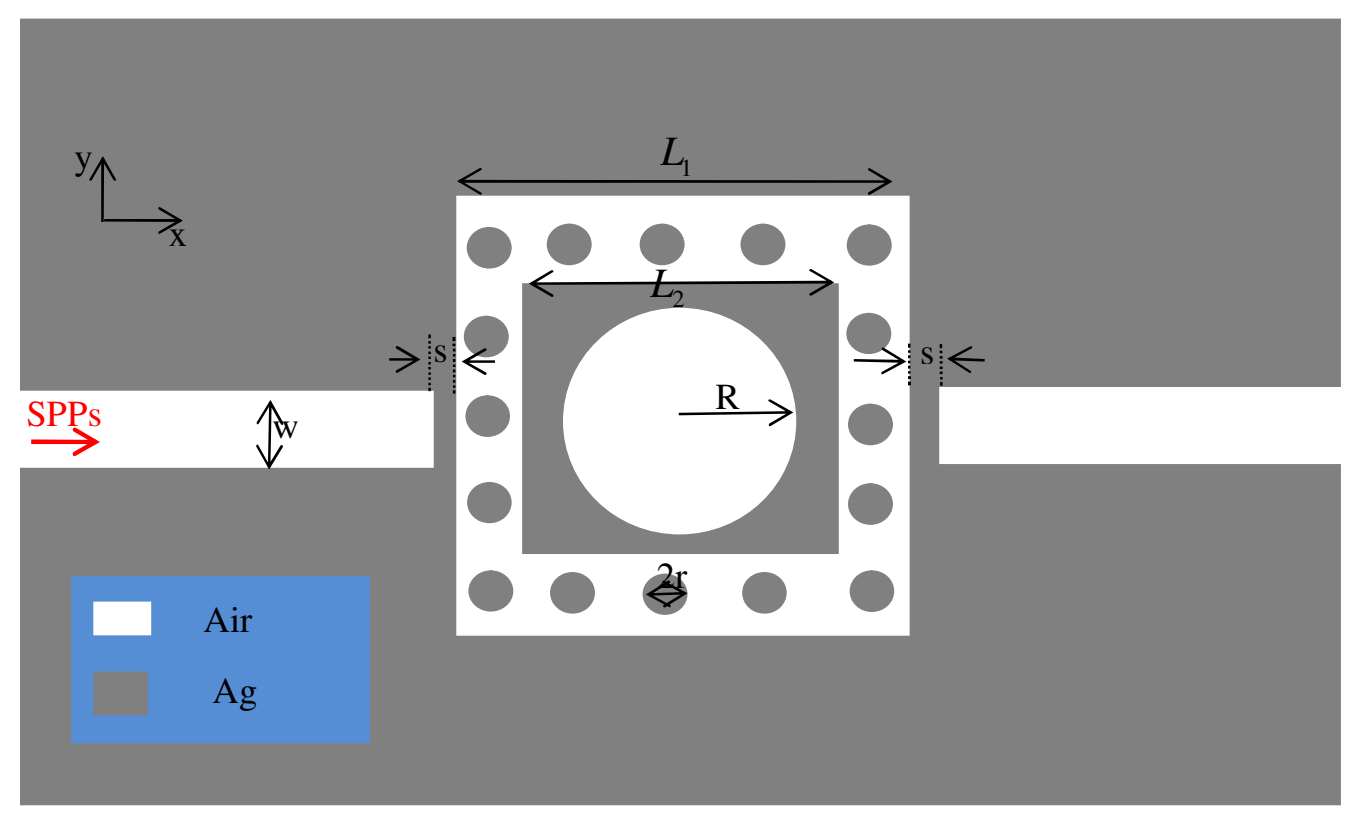

Fig 1. 


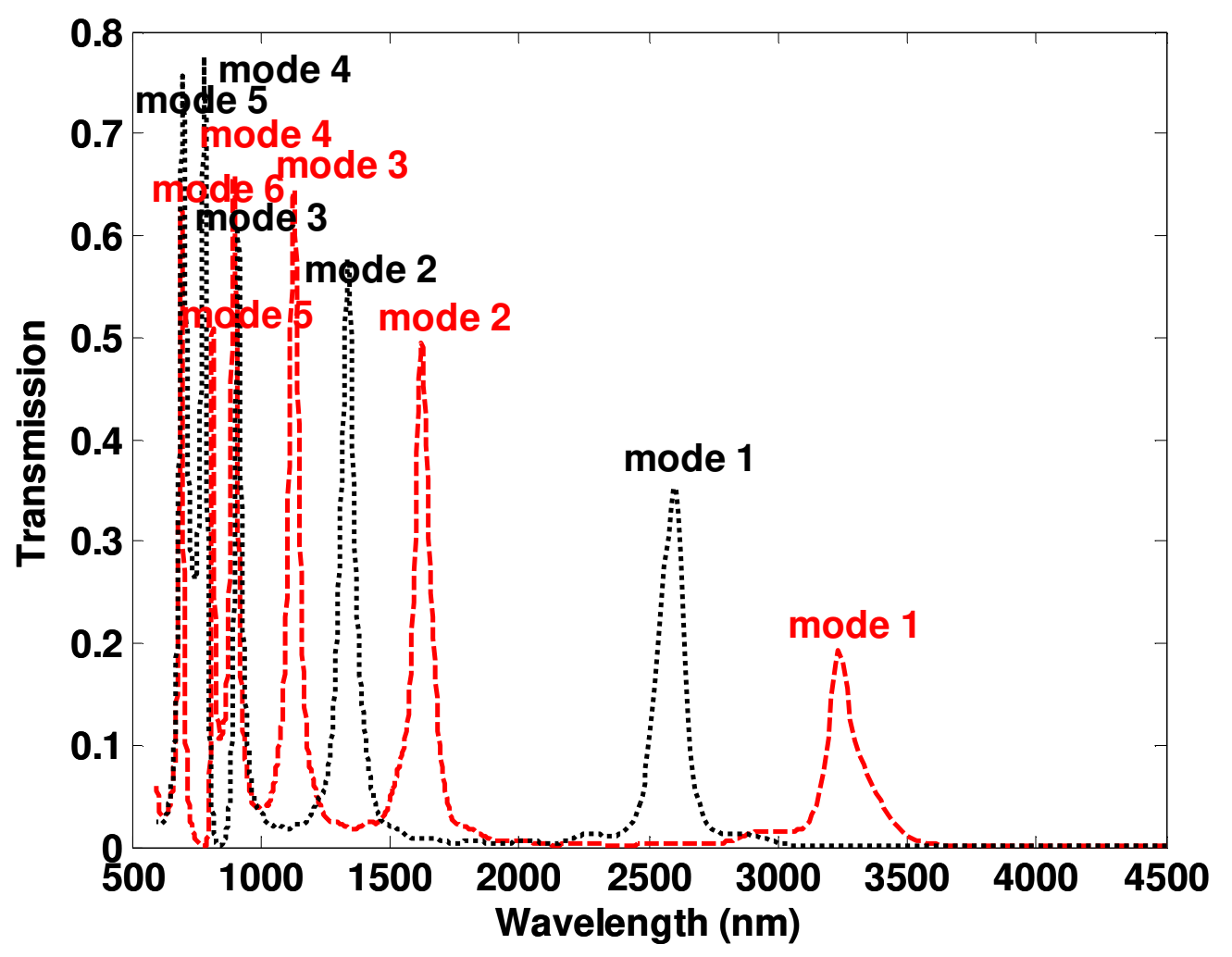

Fig 2. 


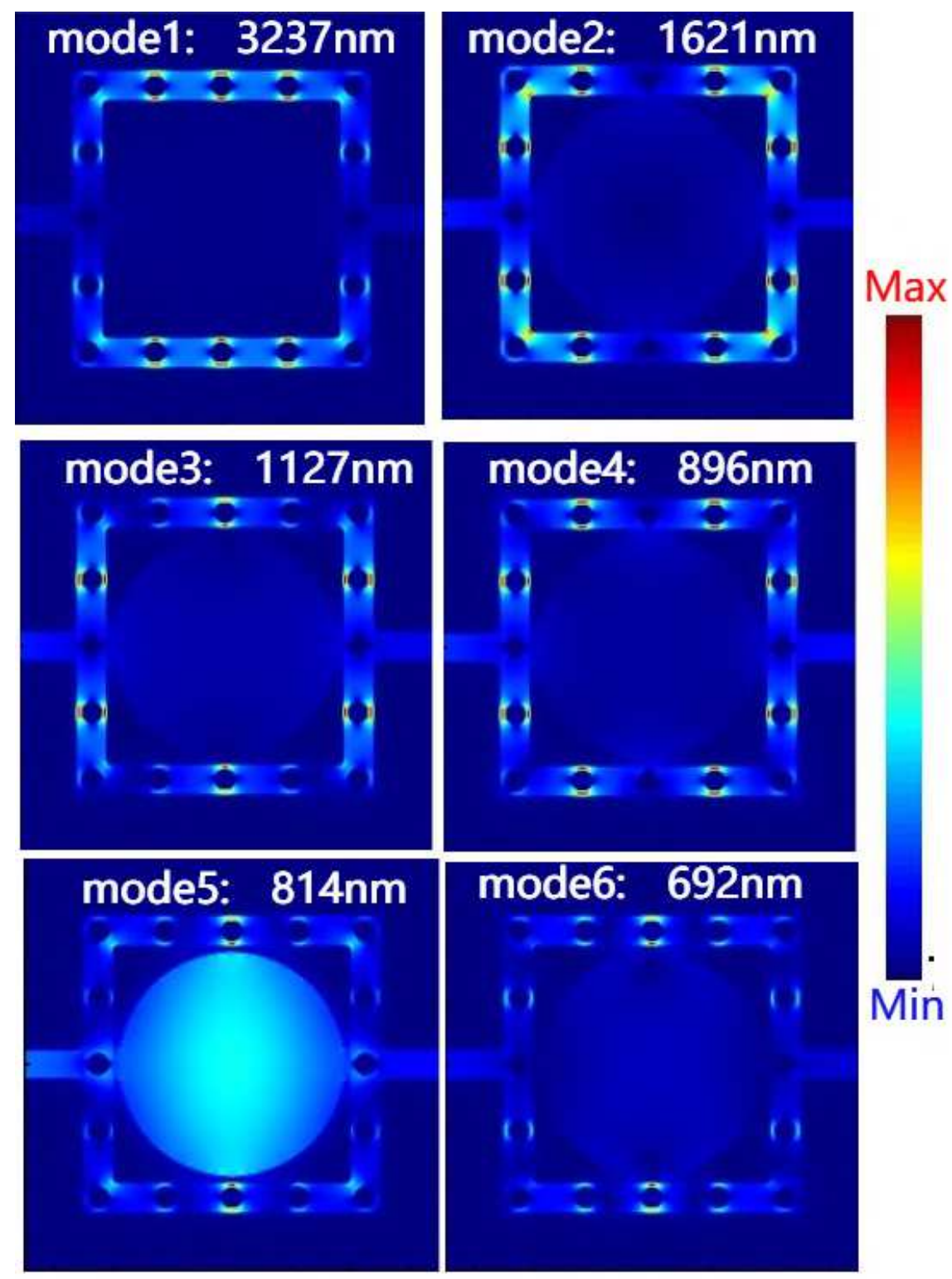

Fig 3. 

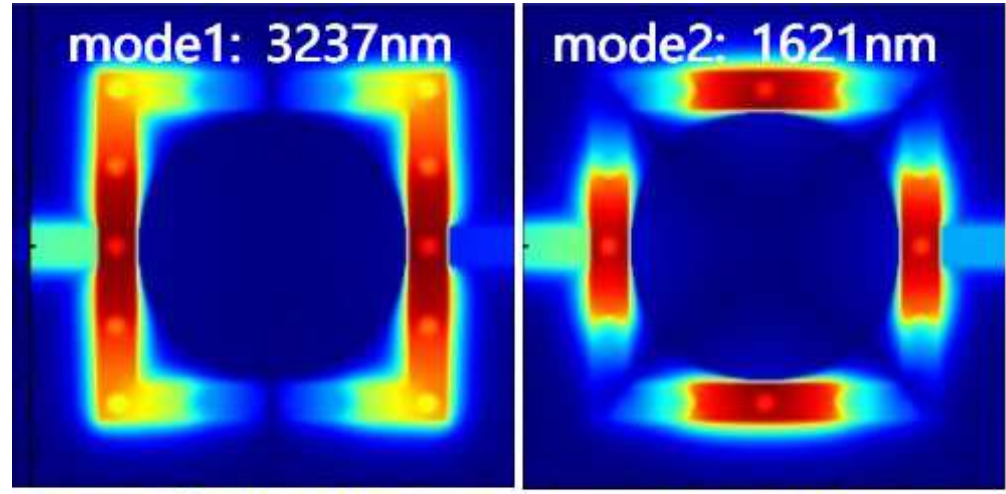

\section{Max}
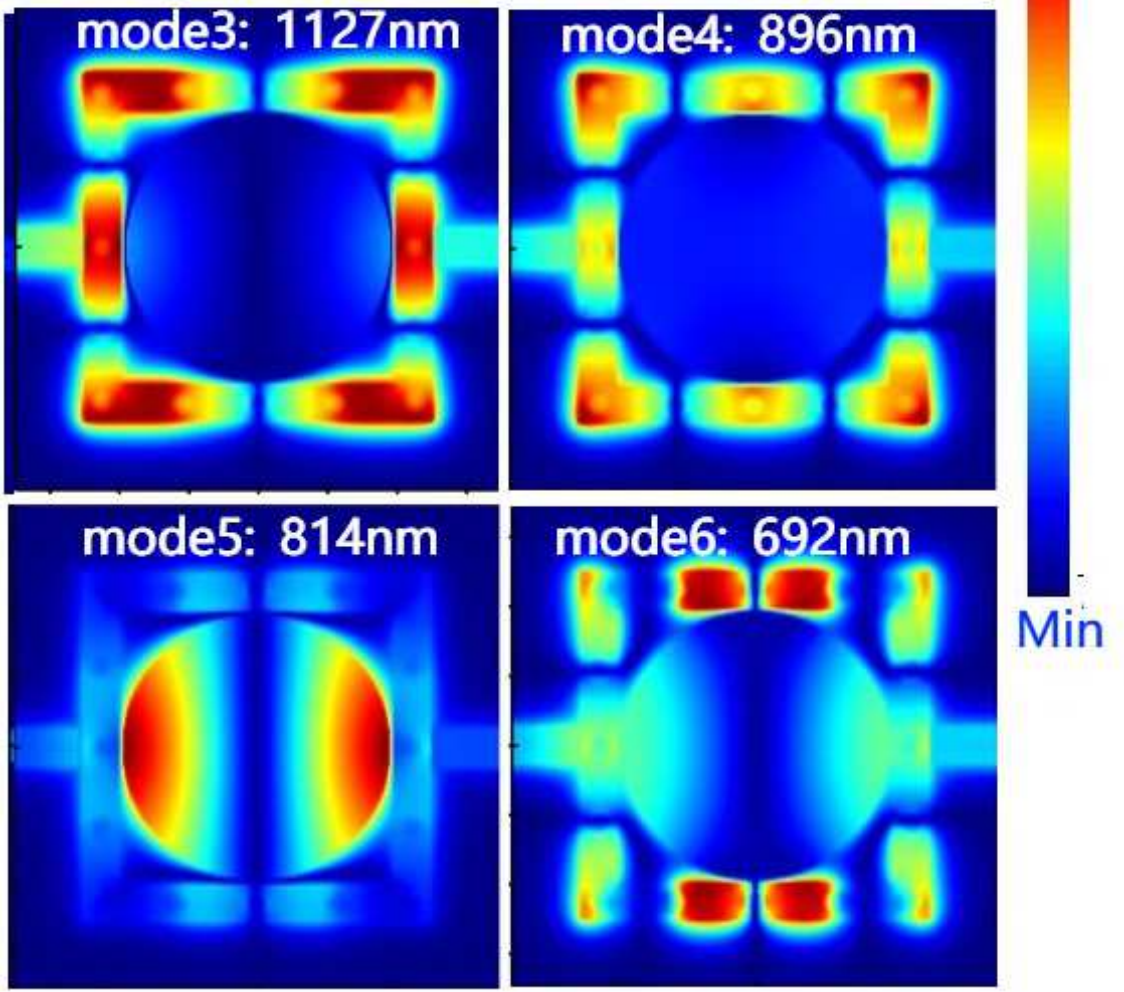

Fig 4. 


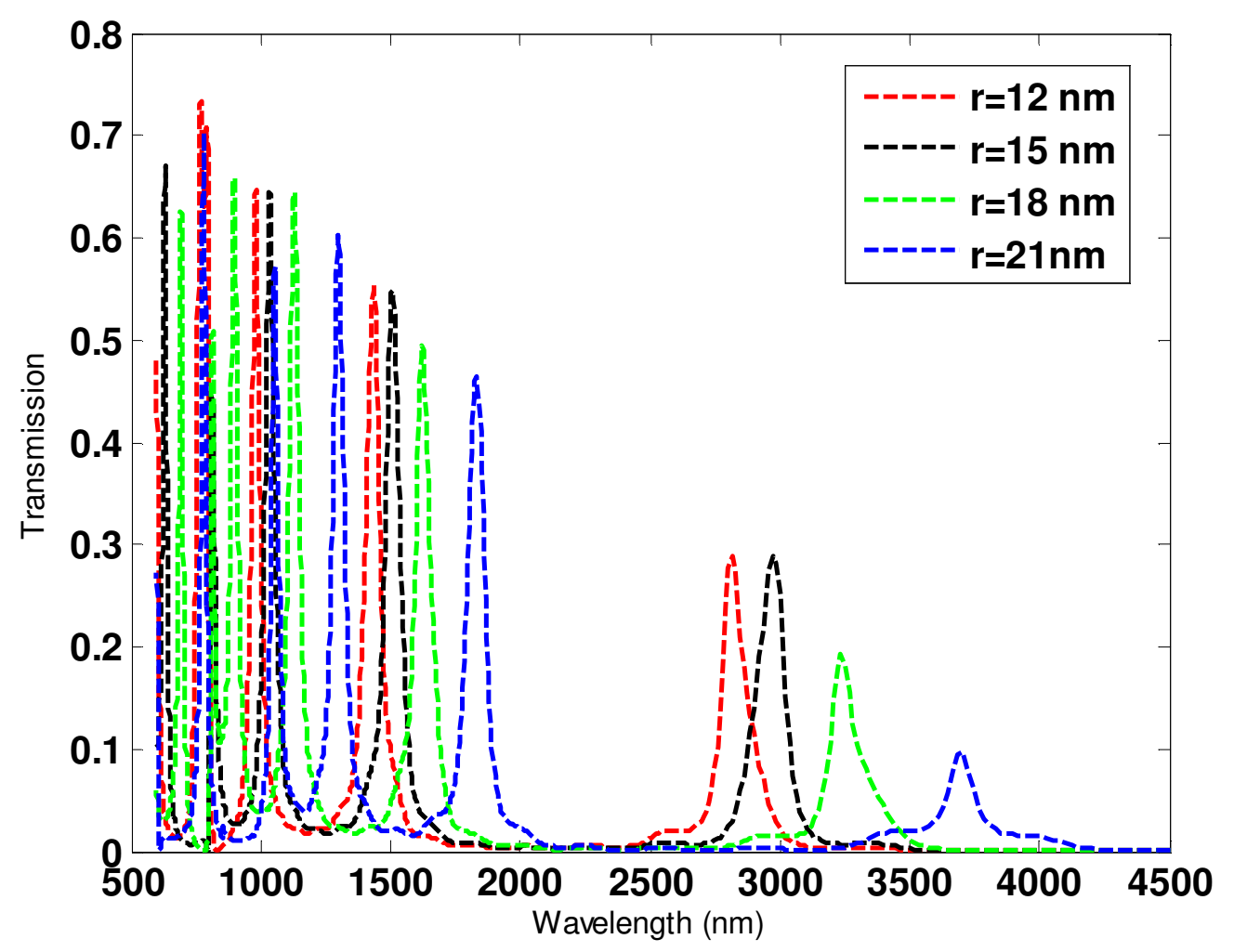

Fig 5. 


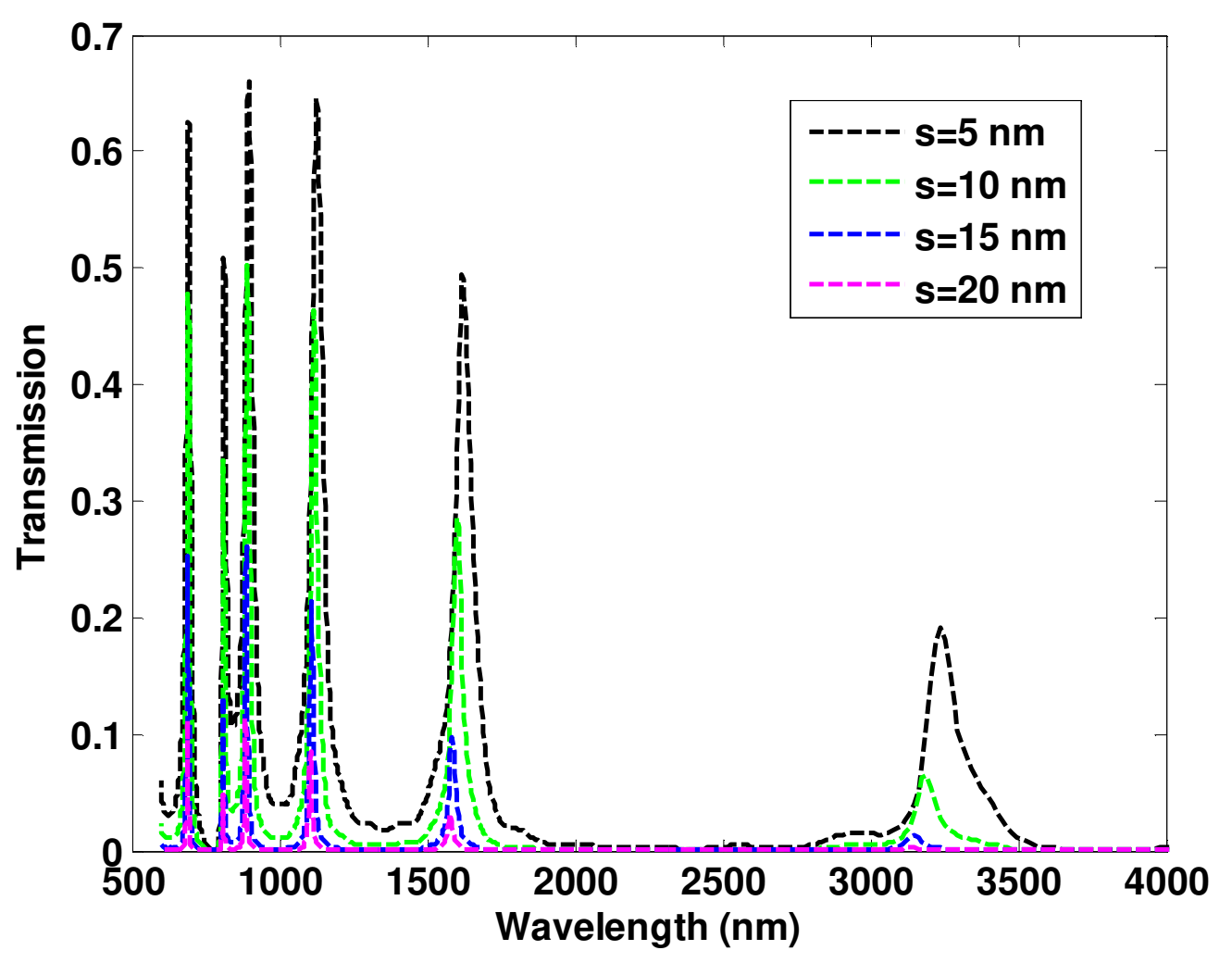

Fig.6. 


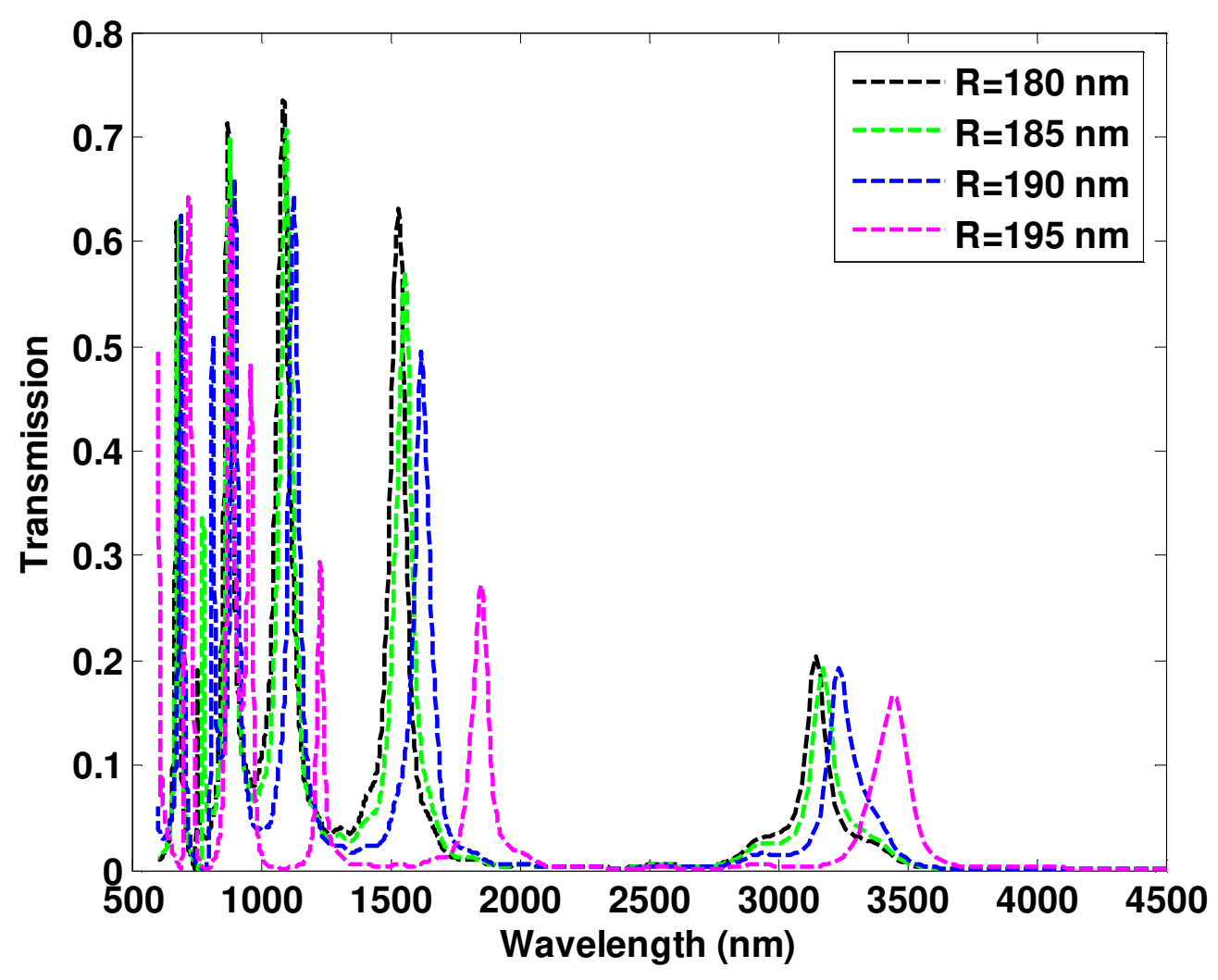

Fig 7. 


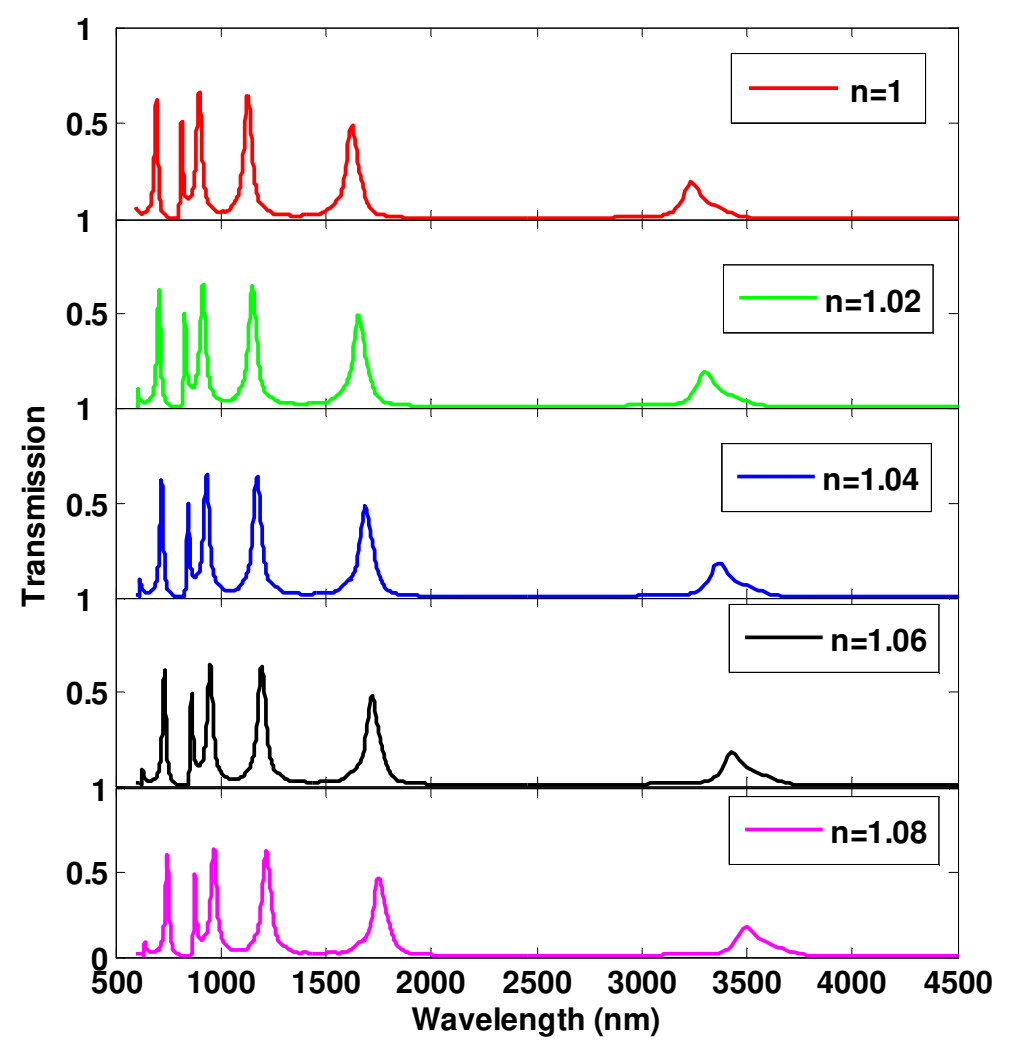

(a)

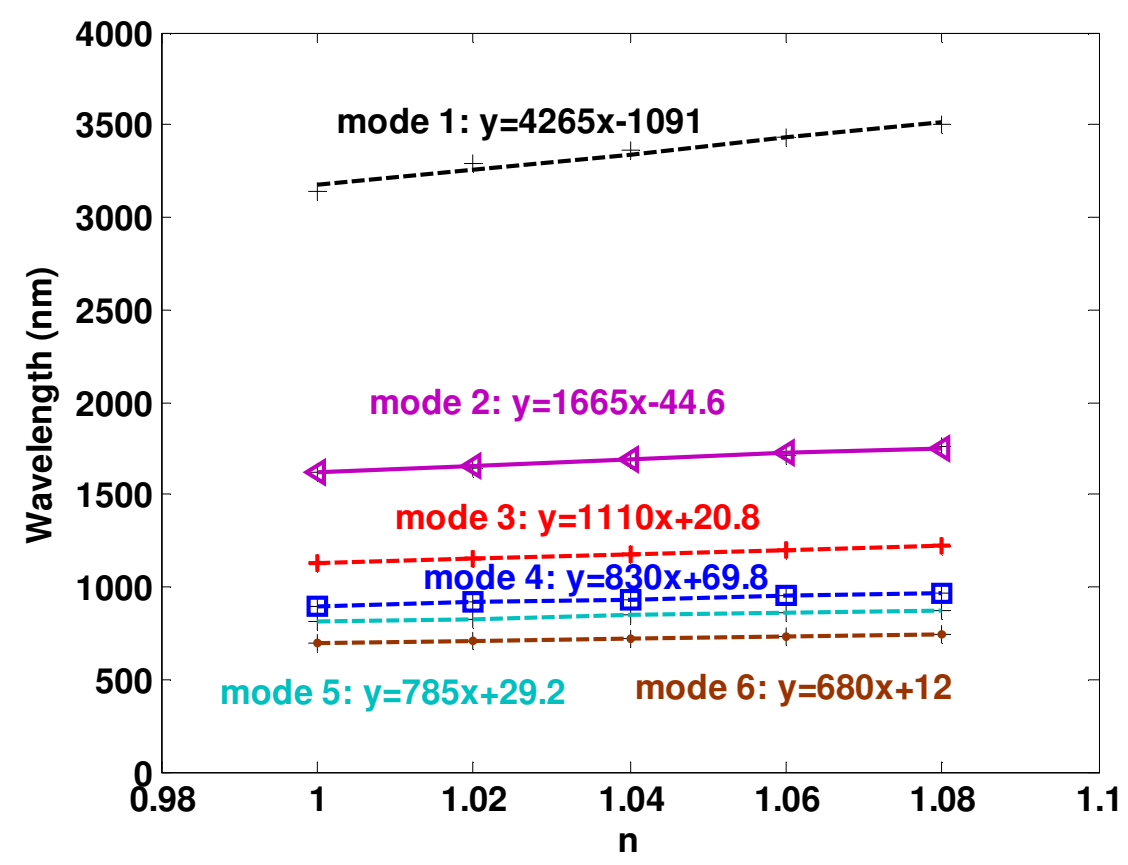

(b)

Fig 8 . 


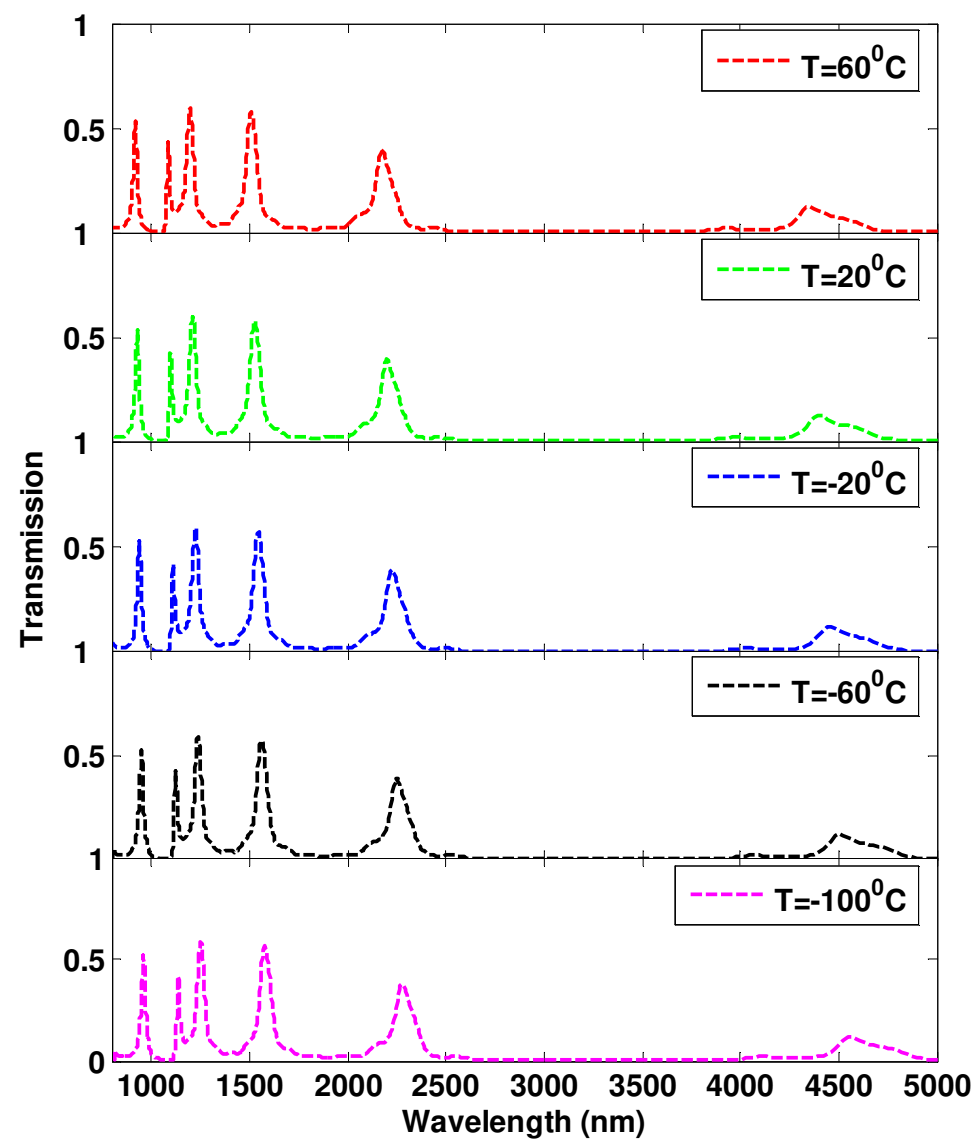

(a)

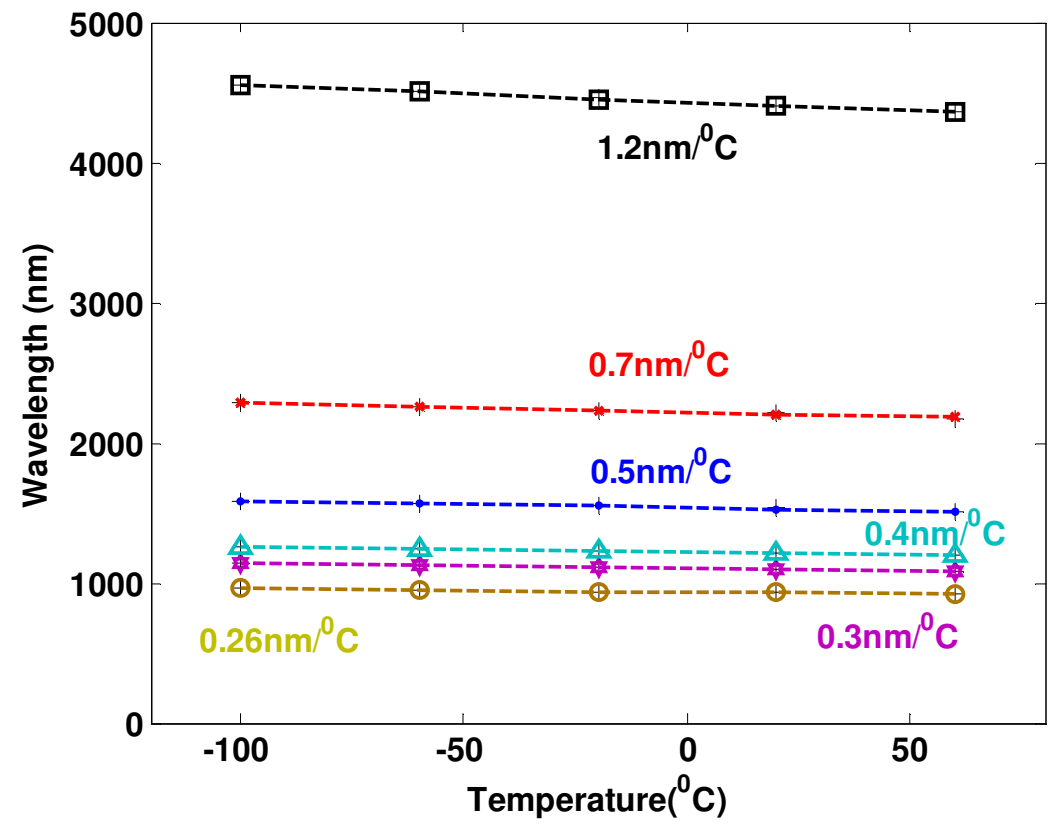

(b) 


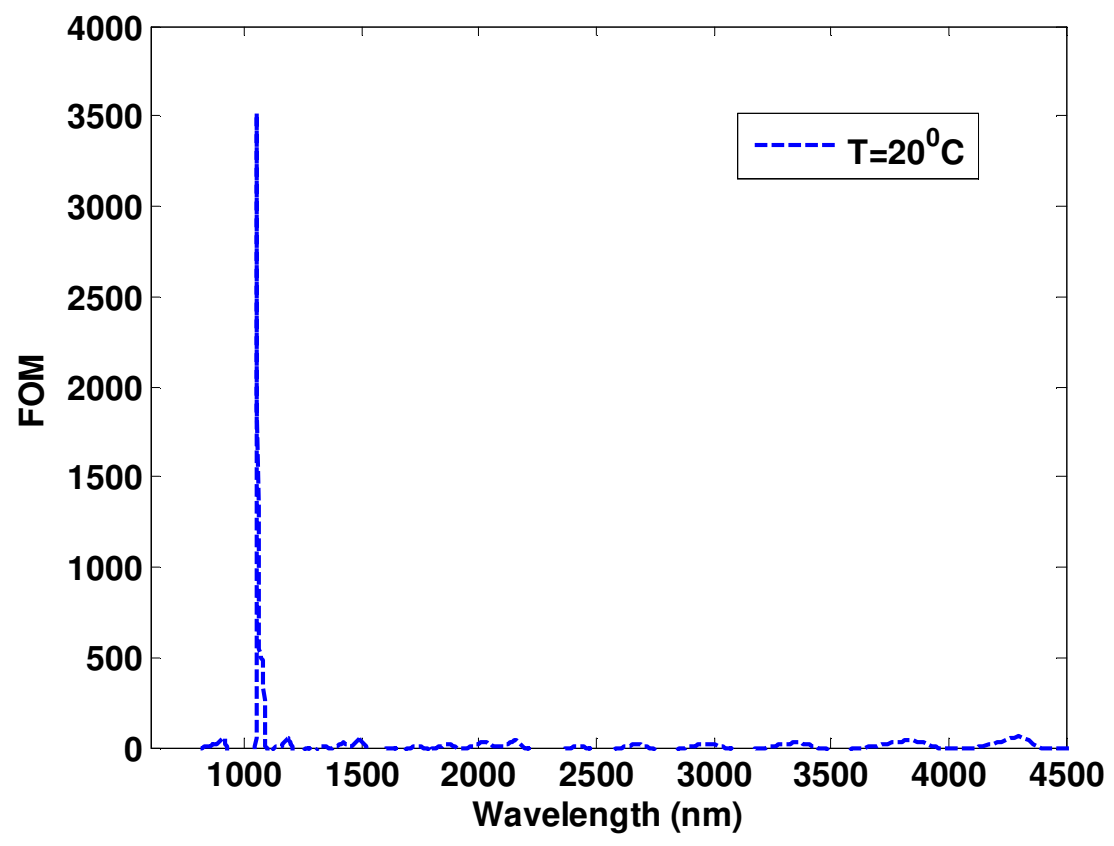

(c)

Fig.9 
Table 1

\begin{tabular}{|l|c|c|c|c|c|c|}
\hline Sensitivity $\left(\mathrm{nm} /{ }^{\circ} \mathrm{C}\right)$ & Mode 1 & Mode 2 & Mode 3 & Mode 4 & Mode 5 & Mode 6 \\
\hline With nanorod defect & 1.2 & 0.7 & 0.5 & 0.4 & 0.3 & 0.26 \\
\hline Without nanorod defect & 0.9 & 0.6 & 0.4 & 0.3 & 0.27 & \\
\hline
\end{tabular}

Table 2

\begin{tabular}{|l|c|c|c|}
\hline \multicolumn{1}{|c|}{ Structure in other work } & Reference & Sensitivity & FOM \\
\hline Nanorod defect coupled square ring resonator & This work & 4265 & 3500 \\
\hline Equilateral triangular resonator with a silver bar & Ref 34 & 923 & 710 \\
\hline Dielectric metal metasurface & Ref 35 & 1800 & 62 \\
\hline Double split ring resonator & Ref 36 & 1260 & 120 \\
\hline Ring resonator coupled to stub & Ref 37 & 2000 & 8600 \\
\hline SPRs/Rod composed of two metallic SRRS and a & Ref 39 & 552 & 920 \\
\hline nanorod & & & \\
\hline
\end{tabular}

